\title{
Statistical analysis of magnetotail fast flows and related magnetic disturbances
}

\author{
Dennis Frühauff and Karl-Heinz Glassmeier \\ Institut für Geophysik und extraterrestrische Physik, Braunschweig, Germany \\ Correspondence to: Dennis Frühauff (d.fruehauff@tu-bs.de)
}

Received: 16 February 2016 - Revised: 16 March 2016 - Accepted: 4 April 2016 - Published: 12 April 2016

\begin{abstract}
This study presents an investigation on the occurrence of fast flows in the magnetotail using the complete available data set of the THEMIS spacecraft for the years 2007 to 2015. The fast flow events (times of enhanced ion velocity) are detected through the use of a velocity criterion, therefore making the resulting database as large as almost 16000 events. First, basic statistical findings concerning velocity distributions, occurrence rates, group structures are presented. Second, Superposed Epoch Analysis is utilized to account for average profiles of selected plasma quantities. The data reveal representative time series in near and far tail of the Earth with typical timescales of the order of 1-2 min, corresponding to scale sizes of $3 R_{\mathrm{E}}$. Last, related magnetic field disturbances are analyzed. It is found that the minimum variance direction is essentially confined to a plane almost perpendicular to the main flow direction while, at the same time, the maximum variance direction is aligned with flow and background field directions. The presentation of the database and first statistical findings will prove useful both as input for magneto-hydrodynamical simulations and theoretical considerations of fast flows.
\end{abstract}

\section{Introduction}

Fast flow events or flow bursts as part of bursty bulk flows (BBFs) (Angelopoulos et al., 1992) play a potentially important role in substorm physics and magnetotail dynamics. According to the outside-in model (see e.g. Ohtani et al., 2004), flow-braking and diversion in the near-Earth region can lead to significant reduction of the cross-tail current (Shiokawa et al., 1997; Keiling et al., 2009) and the formation of the substorm current wedge (Birn and Hesse, 2013; Yao et al., 2012). While global and one-to-one correspondence between fast flows and substorms is not guaranteed, various key substorm signatures could be observed in the predicted order in some events (Baker et al., 2002; Ohtani et al., 1999). Details are also seen in recent simulations (Ebihara and Tanaka, 2015). For a comprehensive review on substorm dynamics please refer to Sergeev et al. (2012); Kepko et al. (2015). Bursty bulk flows are often accompanied by dipolar changes in the magnetic field (Ohtani et al., 2004), which is why they are closely related or even synonymous to dipolarization fronts and dipolarization flux bundles. According to Angelopoulos et al. (1992) a flow burst is a single velocity burst embedded into large-scale structures, series of flow bursts, which are termed bursty bulk flows. Their close relation to various magnetospheric phenomena such as storms and substorms, Pi2 pulsations, and auroral activity makes it worth the effort to study them. Since the late 1980s numerous studies have addressed single case studies, series of events, and statistical characteristics of a great numbers of events. Hayakawa et al. (1982) have performed statistical analysis of fast flows in the neutral sheet using IMP-6 data. Baumjohann et al. (1988) analyzed high-speed ion flows in AMPTE data in the central plasma sheet and plasma sheet boundary layer and found that the average bulk speed is below $100 \mathrm{~km} \mathrm{~s}^{-1}$, therefore agreeing with Huang and Frank (1986). Baumjohann et al. (1989) determined statistical properties of ion fast flows to be of less than 1 min duration, separated by nearly stagnant plasma intervals. Baumjohann et al. (1990) used 8 months of AMPTE data to perform statistical studies in the inner and outer central plasma sheet. Angelopoulos et al. (1992), using AMPTE data as well, created the term bursty bulk flow and found that the half-width of single flow bursts is of the order of $1 \mathrm{~min}$. Angelopoulos et al. (1994) used AMPTE and ISEE2 data to address statistical properties of BBFs and found that they are phenomena of magnetospheric active times while 
single fast flows do not make a substorm. Angelopoulos et al. (1997) found the azimuthal half-scale size to be about $3 R_{\mathrm{E}}$ using Geotail and WIND data. Kepko et al. (2001) examined the relation to Pi2 pulsations in a series of Geotail events and suggested a linking model of flow bursts and Pi2. Nakamura et al. (2001) performed statistical studies on Geotail data and revisited the plasma bubble with a typical timescale of about 2 min as introduced by Pontius Jr. and Wolf (1990) and Sergeev et al. (1996). Hsu and McPherron (2007) used ISEE data to analyze the relation of plasma flows and Pi2 statistically. Sharma et al. (2008) gave a review on various transient phenomena in the magnetotail, focusing on studies performed by Ohtani et al. (2004) who examined the temporal structure of fast flows in Geotail observations and simulations where it was found that fast flows are often accompanied by dipolar field changes and increases in density. Zhang et al. (2009) used Double Star 1 data for statistics on fieldaligned BBFs. Kim et al. (2010) investigated physical parameters of flow bursts in Cluster data and found that the maximum flow speed is proportional to the density depletion of many events. Runov et al. (2011) performed superposed epoch analysis on dipolarization fronts found in THEMIS data and found the short-time minimum variance direction of dipolarization fronts to be aligned with the background flow. Schödel et al. (2001) found the typical timescale of rapid flux transport events to be of $100 \mathrm{~s}$. Liu et al. (2013) analyzed dipolarization flux bundles together with fast flows found in THEMIS data and compiled a list of several hundreds of events. Recently, Schmid et al. (2015) performed statistical studies on dipolarization fronts and plasma flows in THEMIS data and hypothesize on the development of such events. Yao et al. (2015) used the list of Liu et al. (2013) to try to physically explain the dipolarization feature of fast flows. Runov et al. (2015) showed that the characteristic timescale of the flux transport enhancements does not depend on geocentric distance and is around 40-60 s. Most recently, Wang et al. (2016) found evidence of compressible fluctuations during bursty bulk flows in the plasma sheet.

Since numerous statistical studies have already been performed, the aim of this work is not to reinvent the wheel but rather to provide a more comprehensive database of events using THEMIS data. Partial differences in previous findings further motivate revisiting available databases. Due to the orbital configuration, different science phases, and the uplift of two of the five spacecraft to a lunar orbit, various events are available in a total of 9 years of operation. Therefore, a comprehensive list of events can be compiled to be input to statistical methods. The goal is to present the database and the first findings on statistical properties. Detailed interpretation and further analysis will be postponed to future studies. Still, the results will be very useful for both theoretical considerations as well as computer simulations.

The paper is organized as follows: Sect. 2 presents the database and event selection method. Section 3 treats main statistical findings concerning velocity distributions, activity relations, group structure, while Sect. 4 treats results of superposed epoch analysis on selected plasma quantities. Section 5 is concerned with properties of magnetic field variations during the fast flows, and Sect. 6 will summarize the results.

\section{Data and orbital distribution}

The Time History of Events and Macroscale Interactions During Substorms (THEMIS) mission consists of five identical spacecraft launched in 2007 (Angelopoulos, 2008) to study transient and spatial characteristics of substorm-related phenomena in Earth's magnetosphere. The orbital configuration is such that between 2007 and 2011 all probes were situated $8-30 R_{\mathrm{E}}$ from Earth in the ecliptic plane with their apogee being changed continuously to provide $3-4$ months of dayside, dusk, tail, and dawn science phases. In 2011 two of the probes were brought into a lunar orbit, therefore orbiting Earth at distances of about 40-60 $R_{\mathrm{E}}$ (Angelopoulos, 2011). The data include all available science data years of THEMIS, therefore spanning the years 2007-2015 to be as comprehensive as possible. For this study data from the electrostatic analyzers (ESA) for electron and ion plasma $(\leq 30 \mathrm{keV})(\mathrm{Mc}-$ Fadden et al., 2008), and the fluxgate magnetometer (FGM) (Auster et al., 2008) are used. The ESA instrument provides plasma parameters, such as densities, temperatures, and bulk velocities at $\approx 3 \mathrm{~s}$ resolution. Vector magnetic field data are available at $\approx 0.25 \mathrm{~s}$ resolution for most of the selected intervals. Magnetospheric activity indices were obtained from the World Data Center in Kyoto, Japan.

To identify fast flow events in the Earth's magnetotail region, first, a tail box is defined for a total data interval spanning the years 2007-2014 using $X_{\mathrm{GSM}} \leq-8 R_{\mathrm{E}},\left|Y_{\mathrm{GSM}}\right| \leq$ $15 R_{\mathrm{E}}$, and $\left|Z_{\mathrm{GSM}}\right| \leq 10 R_{\mathrm{E}}$. The last of the three orbit criteria is usually not exceeded due to the orbital configuration of the spacecraft.

In a second step, the available ion bulk flow velocity is retrieved from the THEMIS database in Geocentric Solar Magnetic coordinates (GSM). Following earlier studies (see, e.g., Baumjohann et al., 1990 or Angelopoulos et al., 1992) the data are then block averaged to $5 \mathrm{~s}$ resolution, i.e., due to the data availability each block consists of one sample for most of the time. To further improve the detection performance, the data are smoothed using a pseudo-Gaussian sliding average with an effective window length of 13 samples. By doing this, sub-minute variations are canceled while the overall fast flow signatures can be kept. The minimum event separation in the total database will therefore be of the order of $1 \mathrm{~min}$. This step is only used to find relevant events and characterizing their mean parameters. In the detailed analysis, no smoothing is performed, unless specified otherwise.

For the detection of fast flows a simple criterion on the GSM- $X$ component of the ion velocity is applied. Unlike usual approaches, we only require $V_{X} \geq 200 \mathrm{~km} \mathrm{~s}^{-1}$, there- 


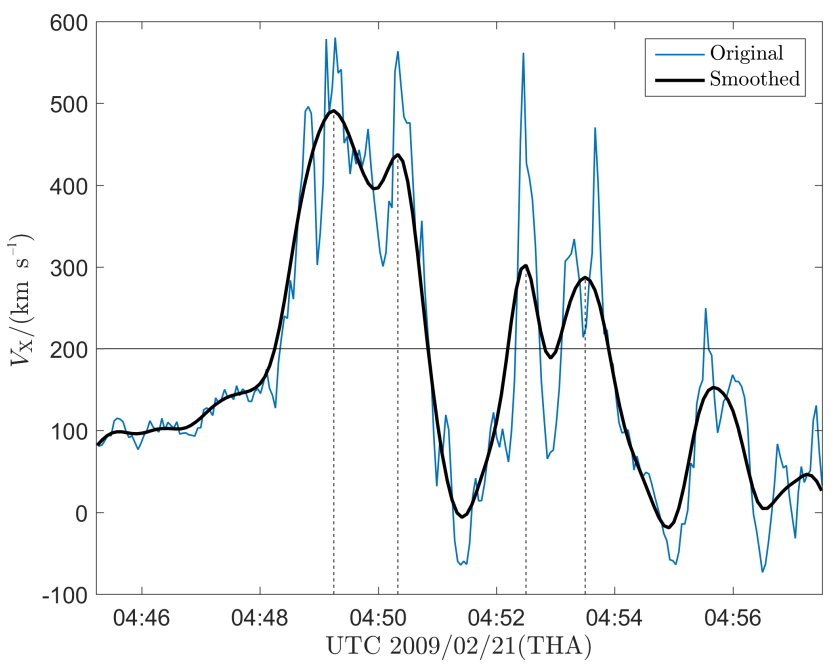

Figure 1. Original example GSM ion velocity time series and smoothed and averaged reference time series for event detection. Detected events are marked with vertical dashed lines. The velocity threshold is at $200 \mathrm{~km} \mathrm{~s}^{-1}$.

fore (a) making the initial database large by setting the threshold well - but not more than necessary - above average bulk flow, and (b) selecting only earthward propagating events in this step. The reduction to events with higher velocities can easily be done after the whole database has been processed.

An example time series is shown in Fig. 1. In this interval four prominent fast flow events (defined as peak velocities in flow intervals exceeding the threshold criterion) are detected, while at the same time a possible event around 04:56 UTC is discarded, because of the very short lifetime of the original velocity peak. While taken by itself, this particular event resembles its preceding neighbors, it is not classified as a fast flow, just by the definition of the threshold criterion. The strength of this approach does not lie in a sophisticated detection algorithm but in the available amount of data spanning a total of almost 9 years on five identical spacecraft.

Figure 2 shows the resulting orbital distribution of the events found in the data set. Of a total of 15695 events, only 523 lie in the GSM $-X$ interval between -40 and $-20 R_{\mathrm{E}}$. We have therefore decided to group all events into those being in a Downtail Region (DT, 9429 events) beyond $-40 R_{\mathrm{E}}$, and those lying within $-20 R_{\mathrm{E}}$, consequently termed Near-Earth Region (NE, 5752 events). For the near-earth region, most of the events are concentrated at around $-10 R_{\mathrm{E}}$ while downtail the distribution appears broader. The obvious bimodal nature of the near-Earth distribution is entirely accredited to the spacecraft's orbital configuration.

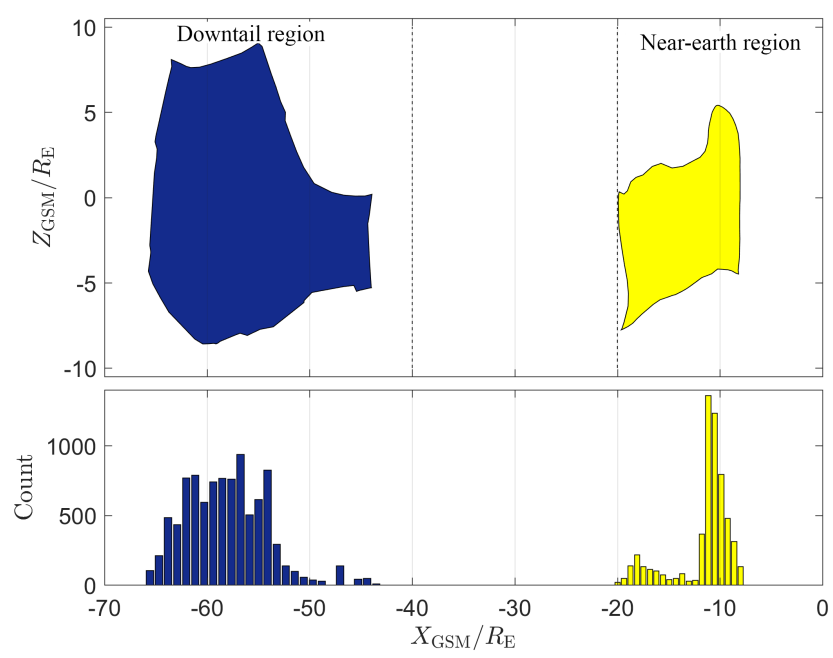

Figure 2. Orbital distribution of the events found in the data set. Due to the bimodal nature, the data are grouped into near-earth (yellow) and downtail (blue) regions. The color code will be kept throughout this paper.

\section{Statistical properties of fast flows in the magnetotail}

\subsection{Velocity distribution}

The peak velocity distribution for all three components of the two regions is shown in Fig. 3. Although the number of events in the two regions is not equal, it is obvious that the $V_{X}$ distributions are essentially equally shaped. Differences can be observed in the $V_{Y}$ and $V_{Z}$ components. The nearEarth distributions are broader with slower events in general. The broadening of the distributions towards Earth indicates the beginning of flow break-up in that region. Additionally, the downtail $V_{Y}$ distribution is slightly shifted towards negative velocities, with its maximum at about $-11 \mathrm{~km} \mathrm{~s}^{-1}$. This shifting originates in the use of natural GSM coordinates and reflects the aberration of the solar wind from the perpendicular. Using the downtail region as a proxy for solar wind aberration, the peak values of the $V_{X}$ and $V_{Y}$ distributions indicate an average aberration angle of $\alpha \approx-2.5^{\circ}$, being in perfect agreement with Fairfield et al. (1996) who found an average of $2.5^{\circ}$ solar wind aberration toward dawn using IMP-8 data.

Taking this into account, the average magnetotail GSM$X$ fast flow is not biased towards perpendicular directions, with peak velocities between 200 and $300 \mathrm{~km} \mathrm{~s}^{-1}$ in GSM-X direction. In other studies, the orbital distribution is usually divided into corresponding magnetotail regions, such as inner and/or outer central plasma sheet, plasma sheet boundary layer, and lobes, therefore distinguishing different $Z_{\mathrm{GSM}}$ regions (Baumjohann et al., 1988, 1990; Angelopoulos et al., 1994). After dividing the current database into $Z_{\mathrm{GSM}}$ layers, no significant differences in the corresponding velocity functions are found. Therefore, no difference is made between 

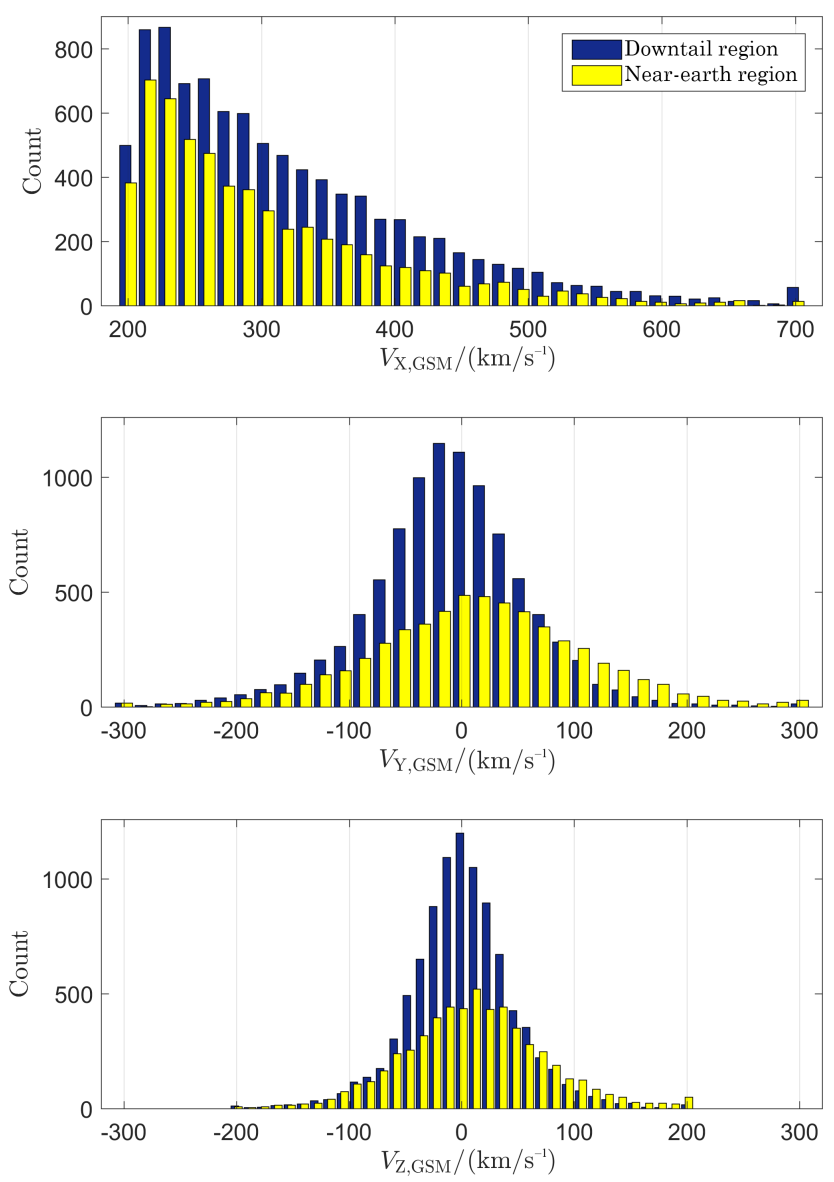

Figure 3. Velocity distribution of all database events for all three components in the GSM frame.

magnetotail regions with respect to current sheet distance. It is rather the goal to present average fast flow characteristics in the whole magnetotail box as defined above.

\subsection{Events and magnetospheric activity}

Following earlier studies (Angelopoulos et al., 1992; Baumjohann et al., 1990), the occurrence rate of fast flows is compared to the AE index as a proxy for magnetospheric activity (Davis and Sugiura, 1966). Angelopoulos et al. (1992) found that $90 \%$ of the detected bursty bulk flows occurred during magnetospheric active times, which they defined as featuring $\mathrm{AE} \geq 100 \mathrm{nT}$. For this work, Fig. 4 displays the corresponding histogram of $\mathrm{AE}$ index and fast flow occurrence. On average, $63 \%$ of all fast flows can be related to an active magnetosphere. It is noteworthy here that no apparent relation has been found between magnetospheric activity and peak velocity of the fast flows. Thus, all events contribute to this histogram. As a consequence, a good portion of all fast flows are found during magnetospheric quiet times, possibly agreeing with Angelopoulos et al. (1994) that a single event does not cause a substorm.

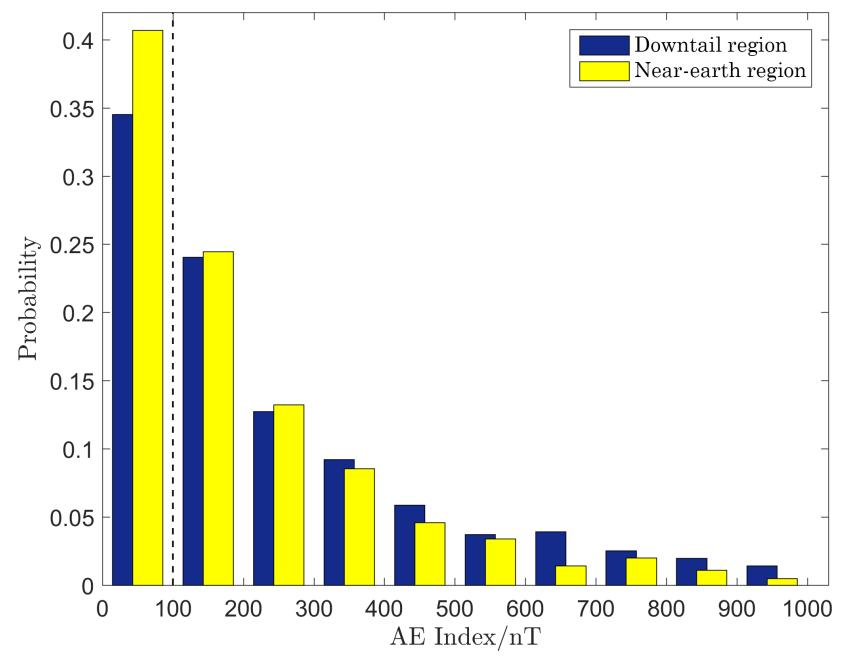

Figure 4. Relation of Fast Flow events and their respective AE index at event time. Events above $100 \mathrm{nT}$ are considered active times.

\subsection{Group structure of fast flows}

Bursty bulk flows are expected to be groups of fast flow events with durations in the order of tens of minutes (Angelopoulos et al., 1992). To examine the group size here, the database has been searched for successive fast flow events that are not separated by more than 10 min each (Angelopoulos et al., 1997). Figure 5 shows the histogram of the resulting group size distribution. Unlike before, the two distributions for downtail and near-Earth region show significant differences. For the near-Earth events, more than $50 \%$ of all fast flows appear as single events. At the same time, their maximum group size rarely exceeds five. On the other hand, only about one third of the downtail events are single fast flows, the group size often reaching 10 or more fast flow events tied together. Two different explanations for these differences can be suggested at this point: (1) the two distributions represent structures generated by two different processes in the magnetotail (or, at least, similar processes taking place in two different tail regimes), that tend to produce single events, or groups of events, respectively; (2) the two distributions are created from one single process in the downtail region that creates groups of events undergoing a converging process into a single bulk flow on their way towards near-Earth and flow braking regions. The latter will also reduce the peak velocity and is likely to remove some events from the statistics. Since our threshold velocity is low, the effect of this systematic error is not changing the global appearance of the distributions.

\section{Superposed epoch analysis of plasma quantities}

Having a database of 15000 events, it is favorable to look for recurring patterns in the data. For the purpose of finding a typical flow burst signature superposed epoch analysis 


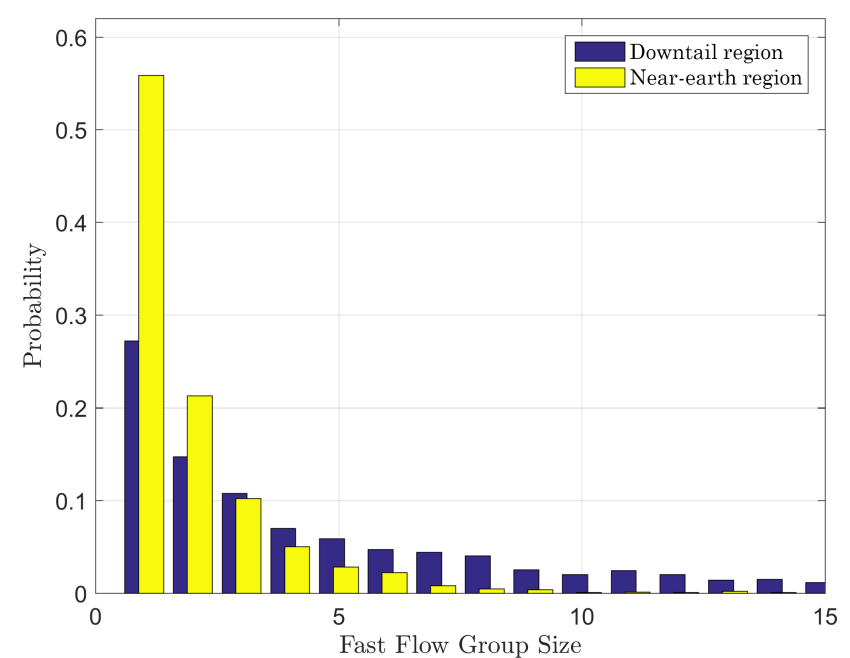

Figure 5. Occurrence of groups in the database. Successive events are considered to belong together when their separation time does not exceed $10 \mathrm{~min}$.

(SEA) is applied to the data (Chree, 1913). This simple technique finds the average or median profile of time series, when the key time, i.e., the time of a defined feature in the event, has been properly defined. While usually the key time for fast flows is defined by using magnetic field data (Ohtani et al., 2004; Yao et al., 2015), here, the peak in $V_{X}$ serves as the key time proxy. Therefore, each peak in the data is treated as a single event constituting to the analysis. Figure 6 displays the results of SEA for various plasma quantities divided in near-Earth and downtail events. The median value for every time step as well as the upper and lower quartiles are shown. In case of density and magnetic field, only the absolute differences to background values were used, to emphasize features in the variations. Various observations can be made at this point: first, the velocity component of the fast flow shows a very prominent signature both for near-Earth and downtail regions. In the first case, a distinct burst-like structure can be observed with very sharp drop-offs to both sides of the event. In case of the downtail events the signature is similar, while the average background flow seems to be much faster. It can be observed that the downtail velocity time series is above our threshold criterion for most of the time. This is solely an offset arising from neighboring events shifting the distribution. From Fig. 5 it can be seen that the downtail events are more likely to occur in groups. Therefore, lifting of the shoulders of the velocity profile is an expected effect. To reveal the unbiased pattern, please refer to Fig. 6 and the description below. Using the velocity profiles to estimate the duration of the fast flow events, one arrives at transit times of $T_{\mathrm{NE}} \approx 100 \mathrm{~s}$ and $T_{\mathrm{DT}} \approx 70 \mathrm{~s}$ agreeing with Baumjohann et al. (1989); Angelopoulos et al. (1992); Nakamura et al. (2001). Together with associated mean velocities of about $\bar{V}_{\mathrm{NE}} \approx 204 \mathrm{~km} \mathrm{~s}^{-1}$ and $\bar{V}_{\mathrm{DT}} \approx 274 \mathrm{~km} \mathrm{~s}^{-1}$ the typical scale sizes of the flows are estimated to be $L_{\mathrm{NE}} \approx 3.2 R_{\mathrm{E}}$

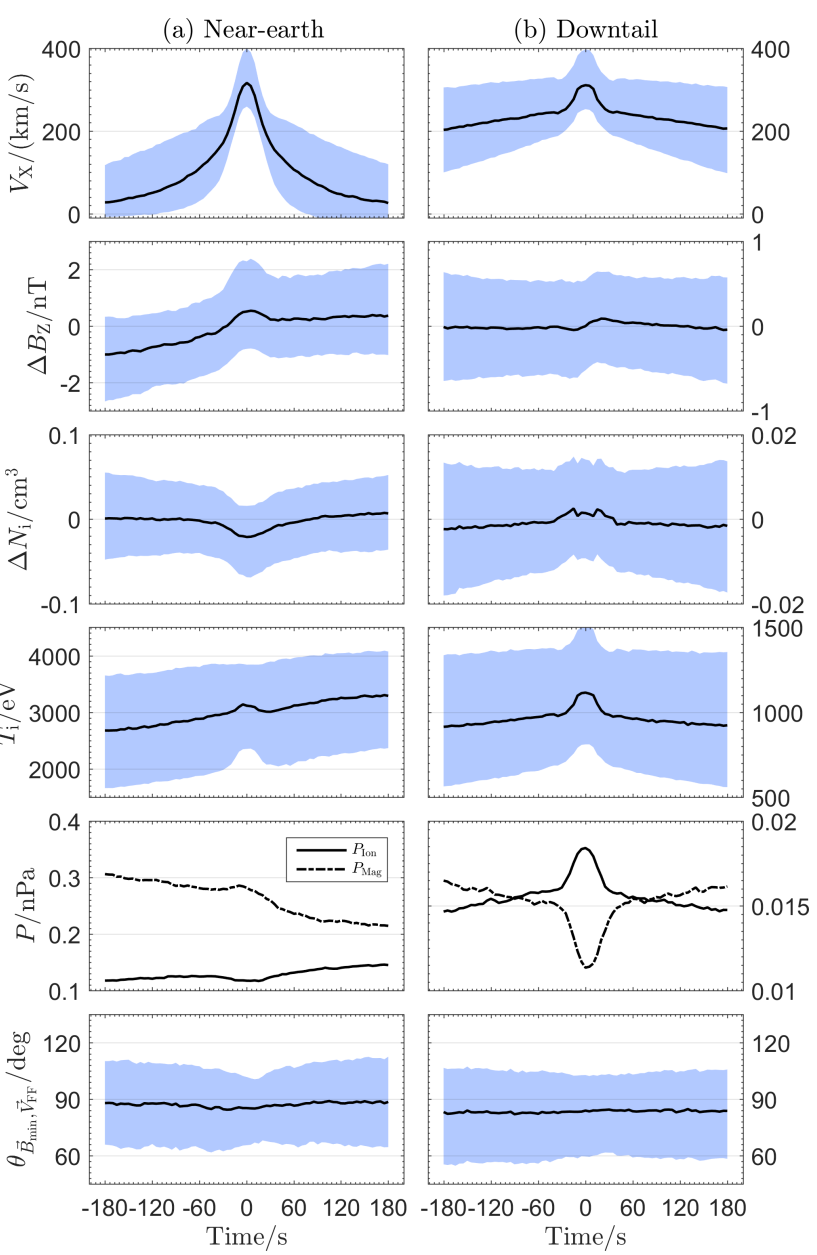

Figure 6. Results of superposed epoch analysis for various plasma quantities. Left column displays near-Earth events, right column shows downtail events. From top to bottom: ion velocity in $X$ direction, magnetic field variation in $Z$ direction, ion density variation, magnetic and ion plasma pressure, angle between flow direction and minimum variance direction of the magnetic field. Time is relative to flow speed maximum. The patched areas indicate the interquartile ranges (not shown for pressure for better visibility).

and $L_{\mathrm{DT}} \approx 3.0 R_{\mathrm{E}}$. Second, for both regions, a dipolar feature in the $Z$ component of the magnetic field can be observed as has already been reported by others (Angelopoulos et al., 1992; Ohtani et al., 2004; Runov et al., 2011; Liu et al., 2013; Yao et al., 2015). Third, the density variation is different for the two regions. While near Earth, the event carries a density depletion, downtail a density enhancement can be observed on average. While it can be seen from the magnetic pressure that the field strength decreases in a similar way, the density enhancement for the downtail region is likely to be a systematic error arising from plasma sheet expansion, i.e., the probe moving from the underpopulated lobes towards plasma sheet periphery. Fourth, for both regions the fast flow carries a temperature enhancement. Fifth, plasma and magnetic pressure indicate total force balance for both 

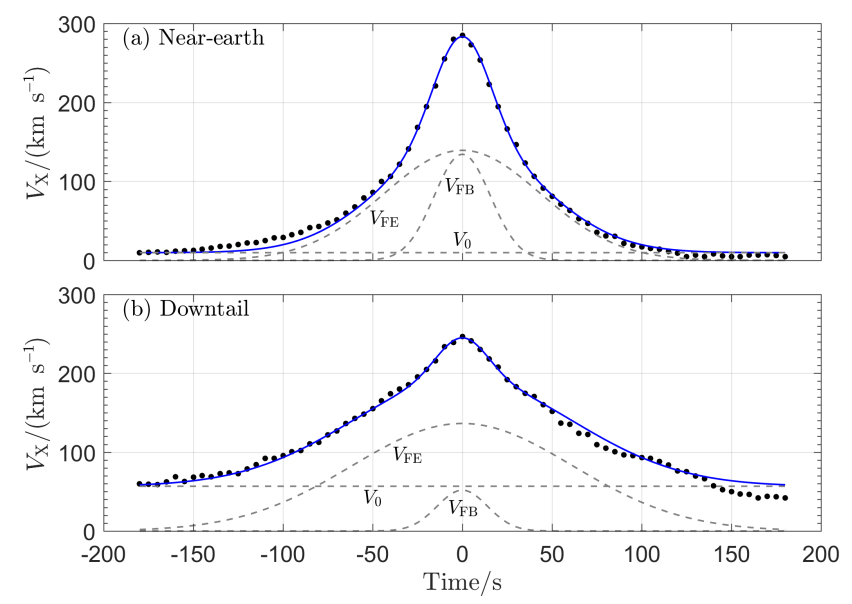

Figure 7. Constituents to fast flow events for (a) near-Earth and (b) downtail events. Data points of SEA (black), resulting least squares fit (blue, solid) and their components (dashed).

regions, indicating no intrinsic expansion or compression of the whole structure. Sixth, the angle between minimum variance direction of magnetic field and main flow direction is a rather constant quantity of almost $90^{\circ}$ for the whole time of the event. Since in the next section minimum variance analysis (MVA) will be applied to magnetic field and velocity data, this pre-justifies the non-criticality of the chosen MVA time interval below.

Having a look at the velocity profiles again, the question arises of how such profiles can be modeled (e.g., to be used as input to numerical simulations). While on first sight it resembles a Gaussian impulse function, the flanks of the profile appear too soft. To quantify such an impulse, near-Earth and downtail events are divided according to Fig. 5, i.e., the group distribution. Now, only isolated events that are not surrounded by additional fast flows within $10 \mathrm{~min}$ on each side are selected and superposed epoch analysis is again performed. The exclusion of groups of events prevents the flanks of the impulse being uplifted by the averaging procedure. Still, to account for any left over smooth slope on each side of the events center, it is assumed that the whole event can be represented by an equivalent model of constituents consisting of a constant offset, $V_{0}$, an enclosing flow enhancement, $V_{\mathrm{FE}}$, and the actual flow burst event, $F_{\mathrm{FB}}$ :

$$
\begin{aligned}
V(t) & =V_{0}+V_{\mathrm{FE}}+V_{\mathrm{FB}} \\
& =V_{0}+\hat{V}_{\mathrm{FE}} \exp \left(-\frac{t^{2}}{2 \sigma_{\mathrm{FE}}^{2}}\right)+\hat{V}_{\mathrm{FB}} \exp \left(-\frac{t^{2}}{2 \sigma_{\mathrm{FB}}^{2}}\right),
\end{aligned}
$$

with time, $t$, peak velocities, $V_{i}$, time constants, $\sigma_{i}$, and imposing $\sigma_{\mathrm{FE}}>\sigma_{\mathrm{FB}}$. To compare the different timescales, the full width at tenth maximum will be used as a transit time proxy for the different constituents: $T_{i}=2 \sqrt{2 \ln 10} \sigma_{i}$. The fitting of the parameters to the data is then performed by nonlinear least squares method using a Levenberg-Marquardt al-
Table 1. Fitting parameters for isolated fast flow events. The values represent the $95 \%$ confident intervals.

\begin{tabular}{lrr}
\hline & Near-Earth & Downtail \\
\hline$V_{0}\left(\mathrm{~km} \mathrm{~s}^{-1}\right)$ & {$[7.7,11.9]$} & {$[52.0,61.5]$} \\
$\hat{V}_{\mathrm{FE}}\left(\mathrm{km} \mathrm{s}^{-1}\right)$ & {$[125,153]$} & {$[128.5,144.3]$} \\
$\hat{V}_{\mathrm{FB}}\left(\mathrm{km} \mathrm{s}^{-1}\right)$ & {$[119,149]$} & {$[47,69]$} \\
$T_{\mathrm{FE}}(\mathrm{s})$ & {$[175.1,199.2]$} & {$[244.4,283.9]$} \\
$T_{\mathrm{FB}}(\mathrm{s})$ & {$[61.2,73.6]$} & {$[45.3,75.3]$} \\
\hline
\end{tabular}

gorithm (Marquardt, 1963). The results are displayed in Table 1 and Fig. 7.

Obviously, the data can be represented very well by the model suggested above. In both cases, the two curves consist of a flow enhancement of similar amplitude, while the background flow is very low for the near-Earth events and much faster for the downtail region. The most striking observation is that the two flow burst components (of downtail and near-Earth, respectively), though of different amplitude, show almost the exact same characteristic timescale $(\sim 60 \mathrm{~s})$. It seems natural to interpret this transit time of the order of $1 \mathrm{~min}$ as an intrinsic feature of isolated fast flow events, possibly related to its initiation and/or injection region. Together with the peak velocities, one arrives at approximate sizes of $1.4 R_{\mathrm{E}}$ (near-Earth) and $0.5 R_{\mathrm{E}}$ (downtail) for the isolated flow burst event. Second, the offset components of both regions are quite different. While this might of course be the consequence of the two different tail regimes, it is also very likely to be an artifact of the analysis. Since, further downtail, GSM positions are becoming more inaccurate in representing the magnetotail correctly (see definition of our box above) the larger offset component is expected to stem from greater tail variability in the downtail region.

\section{Properties of related magnetic field disturbances}

To analyze the relation between fast flows and corresponding, i.e., simultaneous magnetic field variations, minimum variance analysis is applied to the vector magnetic field data. Originally used to determine normal directions of thin discontinuity layers (Sonnerup and Cahill, 1967) in situations of single point measurements, it can also be useful to compute wave propagation directions under the assumption of plane waves. For short notes on MVA see Glassmeier et al. (1993). The main results of the analysis are presented below.

\subsection{Minimum variance direction of magnetic field variations relative to flow direction}

While the determination of the minimum variance direction can be associated with the propagation direction of (magneto-hydrodynamic) wave modes it should be noted that propagation direction might not be the correct term to de- 


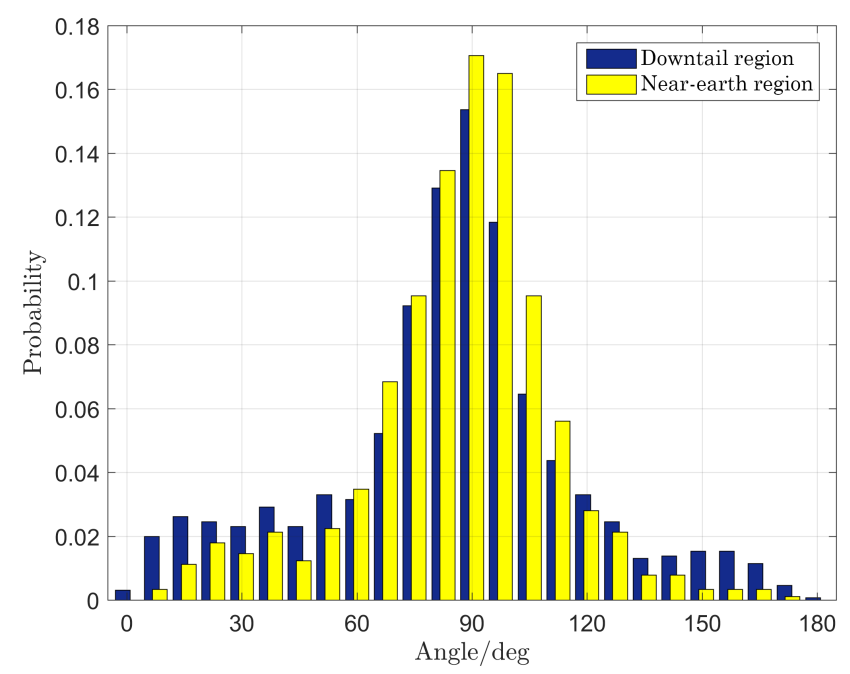

Figure 8. The distribution of the direct angle between flow direction and minimum variance direction of the magnetic field.

scribe the observed phenomena. Thus, the discussion does not intend to interpret this direction as wave vector direction, but rather present the findings made. Still, applying the technique to the velocity data of the events will result in a maximum variance direction, $\boldsymbol{V}_{\max }$, which will serve as a proxy for the main bulk flow direction of the burst. Of course, since the calculation relies on single spacecraft measurements, all retrieved directions are ambiguous in polarity. In case of the flow direction, we already required to select only those events traveling towards Earth as a side condition.

For the analysis of magnetic field and velocity variations $60 \mathrm{~s}$ of data are loaded on either side of the velocity peak time. Usually, the time interval for minimum variance analysis is chosen from magnetic field observations, especially from the dipolarization feature in $B_{Z}$ (see Fig. 6 and, e.g., Runov et al., 2011), and therefore usually of the order of a few seconds. Since, here, velocity data were the starting point and typical timescale was found to be of around 1-2 min, the MVA timescale is chosen accordingly. Therefore, in contrast to most previous studies, we analyze magnetic fluctuations during the total (average) velocity signature. From the observations in Sect. 3 it is obvious that minor variations of the length of this interval do not affect the results significantly.

To ensure that only those events are used where the directions can be well determined, two eigenvalue criteria are imposed on the analysis: (1) for the ratio of median to minimum eigenvalues of magnetic field analysis $\lambda_{\operatorname{med}} / \lambda_{\min } \geq 4$, and (2) for the ratio of maximum to median eigenvalues of velocity analysis $\lambda_{\max } / \lambda_{\text {med }} \geq 4$. For each pair of magnetic field and flow direction vectors, the direct angle, $\Theta_{\mathrm{VB}}$, is computed and constitutes to the histogram shown in Fig. 8.

For downtail and near-Earth regions the two distributions look very similar, showing a very prominent peak at around $90^{\circ}$. Mean values and standard deviations for the two data
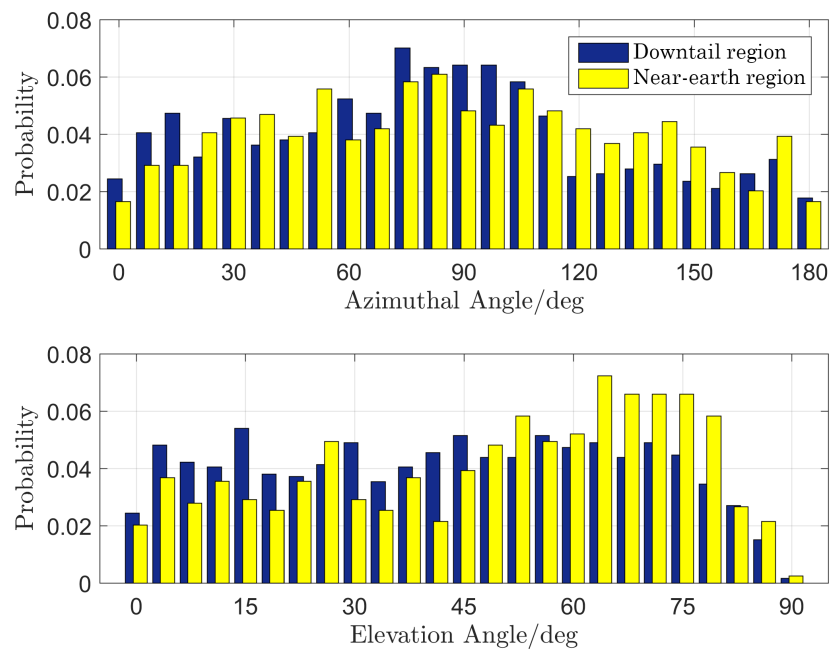

Figure 9. Distribution of orientation angles of the minimum variance direction of magnetic field disturbances referring to GSM coordinates. The azimuthal angle is shown in the upper panel, the elevation can be seen below.

sets are $\theta_{\mathrm{VB}, \mathrm{NE}}=(86 \pm 24)^{\circ}$ and $\theta_{\mathrm{VB}, \mathrm{DT}}=(84 \pm 34)^{\circ}$. Since the distributions are very narrow, this implicates that the minimum variance direction of magnetic field disturbances is essentially confined to a plane perpendicular to the main flow direction.

\subsection{Minimum variance direction of magnetic field variations in the GSM frame}

Figure 9 shows azimuthal and elevation angles for all of the selected minimum variance vectors, $\boldsymbol{B}_{\min }$, to resolve a preferred orientation in the GSM frame. The azimuthal (elevation) angle is defined as the projected angle to the $X-Z$ $(X-Y)$ plane. It should be remembered that the determined direction is ambiguous. Therefore, the distribution has been limited to positive angles. For the azimuthal angle, a slight enhancement towards $90^{\circ}$ can be observed, which is in agreement with the propagation plane idea of the previous subsection. Still, since the main flow direction is not always confined to the GSM- $X$ direction (see Fig. 2), the distribution is very widespread and does not indicate significant absolute directions. As for the elevation angle an enhancement in the probability can be observed for higher elevation angles, which is, again, in agreement with the idea before. This enhancement is more pronounced for the near-Earth data set, probably indicating effects of flow break up and deflection at the dipole field of the Earth. At the same time, the almost absence of elevation angles very close to $90^{\circ}$ is an interesting feature to be observed. Like before, the distribution being widespread leads to the conclusion that the preferred minimum variance direction is only confined to a plane almost perpendicular to the flow direction. 

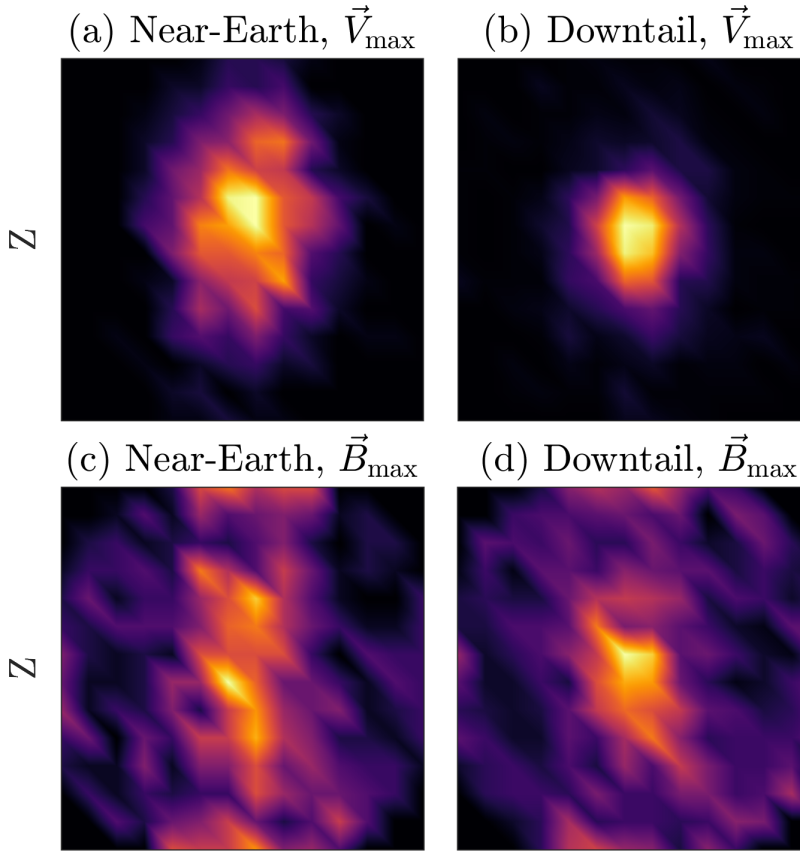

Y
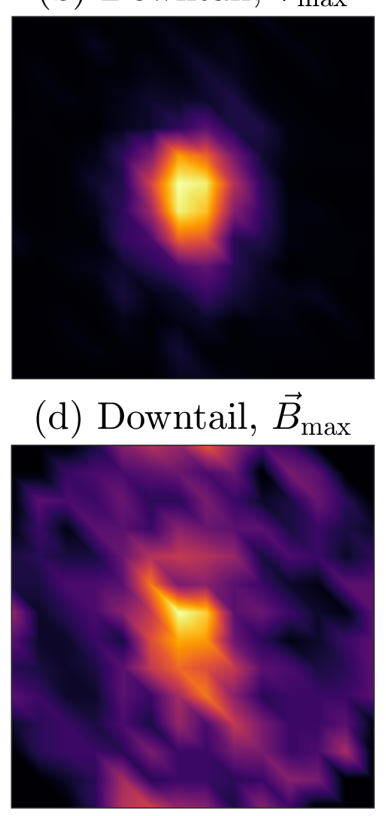

Y

cially for the downtail region, assuming that the background magnetic field is of Harris sheet nature (Harris, 1962), i.e., the background field being parallel to GSM- $X$, this indicates a compressional nature of magnetic field variations for the total flow burst event (see also Wang et al., 2016). Still, there are also many events obviously deviating from the flow direction for which a detailed analysis of the database will be necessary. In both downtail and near-Earth distributions $Z$ appears to be the preferred deviation direction.

Based on the magnetic field measurements alone, and indeed assuming for the moment that the minimum variance direction is an indicator for wave propagation direction, the key characteristics (large amplitude, propagation almost perpendicular to the background field, maximum variance direction aligned with the background field) fit the criteria defined for mirror modes (e.g. Lucek et al., 1999a, b; Schmid et al., 2014). While the aim of this is merely to present selected statistical results, further analysis and interpretation is far beyond the scope of this work.

\section{Summary and conclusion}

Figure 10. (a, b) Vector projection of the maximum variance (flow) direction of the velocity in the GSM frame for near-Earth and downtail regions. (c, d) Vector projection of the maximum variance directions of the magnetic field after flow alignment (normalized units). See text for details.

\subsection{Maximum variance directions of magnetic field and velocity data}

To find out more about the nature of the magnetic field disturbances, the maximum variance directions of the magnetic field and the velocity are compared, again using the technique applied before. The panels $\mathrm{a}$ and $\mathrm{b}$ of Fig. 10 show a projection of all velocity maximum variance vectors, $\boldsymbol{V}_{\max }$, onto the GSM- $Y Z$ plane. The visualization is simplified by computing a two-dimensional histogram with yellow indicating high occurrence rate and black indicating low occurrence rate. From the upper right panel $b$ it can be seen that the main flow direction of the downtail events is well confined to the GSM- $X$ direction and of almost circular shape. On the other hand, the near-Earth events in panel a show a broader variation in the GSM-Z direction, indicating flow break-up and deflection processes.

The two panels below have been produced by computing the maximum variance direction of magnetic field data, $\boldsymbol{B}_{\max }$, and rotating the vectors into a coordinate system in which $\boldsymbol{V}_{\text {max }}$ is perfectly aligned with GSM-X, before the projection density was computed. In such a way, the alignment of $\boldsymbol{V}_{\max }$ and $\boldsymbol{B}_{\max }$ can be qualitatively analyzed. The main observation to be made here is that for a good portion of the available events the maximum magnetic field variations are almost parallel to the flow direction, i.e., $\delta B \| \delta V$. Espe-

The aim of the present work was to investigate the total available THEMIS data to compile a list of flow events in Earth's magnetotail. A total of almost 16000 events are found, many of them embedded in a group of fast flows, so-called bursty bulk flows. As to this date, this is the largest list of fast flow events that has been produced. Due to the orbital distribution of the events, two different regions were defined as nearEarth and downtail region. While many statistical characteristics are similar for the two distributions, some differences were found as well. The velocity distributions are very similar, the average fast flow is directed in GSM- $X$ direction, slightly shifted for the downtail region due to the aberration of the solar wind. Up to two thirds of all fast flow events occur during magnetospheric active times, while more than $50 \%$ appear as single structures not embedded in a series of events. Through the use of superposed epoch analysis, average profiles of selected plasma quantities are found with interesting differences in the density variations for near-Earth and downtail events. While the former carries density depletion, the latter is accompanied by density enhancement on average. Analyzing only isolated fast flow events a characteristic timescale of about $60 \mathrm{~s}$ is found for the bursty component of all events, which is present both in near-Earth and in downtail data. Since the database is very large and is likely to comprise various and in detail very different events, accurate quantification of the average timing relation of different plasma quantities is difficult. Detailed investigation and consistent division of the data into subcategories will yield meaningful results in follow-up publications.

Associated minimum variance directions of magnetic field disturbances seem to be confined to a plane almost perpendicular to the main flow direction. This is a feature that is per- 
sistent for the whole time of the event. While the relative direction is therefore clearly defined, nor preferred orientation can be observed in GSM coordinates. Maximum variance directions of magnetic field and velocity data are aligned for the larger part of the database, i.e., the maximum variance direction of the magnetic field fluctuations is along GSM-X .

The nature of these fluctuations is not understood through observations alone. Especially, it is not clear, whether the inferred minimum variance directions can serve as a proxy for propagation directions, which is a key information necessary to develop a bigger picture of these phenomena.

These findings are contrary to many previous studies on the first look (see, e.g., Ohtani et al., 2004; Runov et al., 2011; Liu et al., 2013). Still, since these studies typically chose the dipolarization feature as keytime and used a short MVA interval for their analysis, any differences are not surprising. First, the timescale of the dipolarization feature is short compared to the timescale of the flow burst and, thus, does not contribute significantly to the interval chosen here. Second, not every flow burst event features a prominent dipolarization feature in $B_{z}$. Third, if present, the time lag between $B_{z}$ flip and maximum flow velocity cannot be assumed constant for several thousand events. Therefore, in Fig. 6 the $\Delta B_{z}$ time series does not show the very prominent behavior of other studies.

We summarize important observations as follows:

- Magnetotail fast flows occur both during magnetospheric active and quiet times.

- While downtail multiple burst events dominate, nearEarth isolated events are observed more frequently.

- Superposed Epoch Analysis reveals mean properties of fast flows in both regions. Characteristic timescales are of the order of $60 \mathrm{~s}$ with associated length scales of $3 R_{\mathrm{E}}$, consistent with previous findings (e.g., Angelopoulos et al., 1997; Schödel et al., 2001; Runov et al., 2015).

- Minimum variance directions of the magnetic field are on average perpendicular to the main flow and background magnetic field directions for the total flow burst timescale.

The presentation of the database and first statistical findings is only the start of detailed investigations of fast flow characteristics in the THEMIS data set. The results of the Superposed Epoch Analysis are certainly useful as input to numerical MHD simulations of magnetotail regions. It would be interesting to see the observational findings concerning the magnetic field variations to be confirmed and understood. To achieve this, theoretical aspects need also to be considered in the future. Therefore, future work will include more detailed investigation of different aspects of the data set with a focus on magnetic field fluctuations, theoretical considerations of the observations, especially concerning current sys- tems, drivers of these structures, questions on possible neutral lines, as well as MHD simulations to reproduce similar features in controlled surroundings.

Acknowledgements. Auroral Electrojet indices for all necessary events were obtained from the World Data Center in Kyoto, Japan. We acknowledge NASA contract NAS5-02099 and V. Angelopoulos for use of data from the THEMIS Mission. Specifically: C. W. Carlson and J. P. McFadden for use of ESA data. This project is financially supported by the German Ministerium für Wirtschaft und Energie and the Deutsches Zentrum für Luft und Raumfahrt under contract $500 \mathrm{C} 1403$.

The topical editor, C. Owen, thanks Z. Yao and one anonymous referee for help in evaluating this paper.

\section{References}

Angelopoulos, V.: The THEMIS Mission, Space Sci. Rev., 141, 534, 2008.

Angelopoulos, V.: The ARTEMIS Mission, Space Sci. Rev., 165, 3-25, 2011.

Angelopoulos, V., Baumjohann, W., Kennel, C. F., Coronti, F. V., Kivelson, M. G., Pellat, R., Walker, R. J., Luehr, H., and Paschmann, G.: Bursty bulk flows in the inner central plasma sheet, J. Geophys. Res., 97, 4027-4039, 1992.

Angelopoulos, V., Kennel, C. F., Coroniti, F. V., Pellat, R., Kivelson, M. G., Walker, R. J., Russell, C. T., Baumjohann, W., Feldman, W. C., and Gosling, J. T.: Statistical characteristics of bursty bulk flow events, J. Geophys. Res., 99, 21257-21280, doi:10.1029/94JA01263, 1994.

Angelopoulos, V., Phan, T. D., Larson, D. E., Mozer, F. S., Lin, R. P., Tsuruda, K., Hayakawa, H., Mukai, T., Kokubun, S., Yamamoto, T., Williams, D. J., McEntire, R. W., Lepping, R. P., Parks, G. K., Brittnacher, M., Germany, G., Spann, J., Singer, H. J., and Yumoto, K.: Magnetotail flow bursts: Association to global magnetospheric circulation, relationship to ionospheric activity and direct evidence for localization, Geophys. Res. Lett., 24, 2271-2274, 1997.

Auster, H. U., Glassmeier, K. H., Magnes, W., Aydogar, O., Baumjohann, W., Constantinescu, D., Fischer, D., Fornacon, K. H., Georgescu, E., Harvey, P., Hillenmaier, O., Kroth, R., Ludlam, M., Narita, Y., Nakamura, R., Okrafka, K., Plaschke, F., Richter, I., Schwarzl, H., Stoll, B., Valavanoglou, A., and Wiedemann, M.: The THEMIS Fluxgate Magnetometer, Space Sci. Rev., 141, 235-264, 2008.

Baker, D. N., Peterson, W. K., Eriksson, S., Li, X., Blake, J. B., Burch, J. L., Daly, P. W., Dunlop, M. W., Korth, A., Donovan, E., Friedel, R., Fritz, T. A., Frey, H. U., Mende, S. B., Roeder, J., and Singer, H. J.: Timing of magnetic reconnection initiation during a global magnetospheric substorm onset, Geophys. Res. Lett., 29, 43-1-43-4, 2002.

Baumjohann, W., Paschmann, G., Sckopke, N., Cattell, C. A., and Carlson, C. W.: Average ion moments in the plasma sheet boundary layer, J. Geophys. Res., 93, 11507-11520, 1988.

Baumjohann, W., Paschmann, G., and Cattell, C. A.: Average plasma properties in the central plasma sheet, J. Geophys. Res., 94, 6597-6606, 1989. 
Baumjohann, W., Paschmann, G., and Luehr, H.: Characteristics of high-speed ion flows in the plasma sheet, J. Geophys. Res., 95, 3801-3809, 1990.

Birn, J. and Hesse, M.: The substorm current wedge in MHD simulations, J, Geophys. Res.-Space, 118, 3364-3376, 2013.

Chree, C.: Some Phenomena of Sunspots and of Terrestrial Magnetism at Kew Observatory, Philos. T. R. Soc. Lond., 212, 75116, 1913.

Davis, T. N. and Sugiura, M.: Auroral electrojet activity index AE and its universal time variations, J. Geophys. Res., 71, 785-801, 1966.

Ebihara, Y. and Tanaka, T.: Substorm simulation: Insight into the mechanisms of initial brightening, J. Geophys. Res.-Space, 120, 7270-7288, 2015.

Fairfield, D. H., Lepping, R. P., Frank, L. A., Ackerson, K. L., Paterson, W. R., Kokubun, S., Yamamoto, T., Tsuruda, K., and Nakamura, M.: Geotail Observations of an Unusual Magnetotail under Very Northward IMF Conditions, J. Geomagn. Geoelectr., 48, 473-487, 1996.

Glassmeier, K.-H., Motschmann, U., Mazelle, C., Neubauer, F. M., Sauer, K., Fuselier, S. A., and Acuna, M. H.: Mirror modes and fast magnetoacoustic waves near the magnetic pileup boundary of comet P/Halley, J. Geophys. Res., 98, 20955-20964, doi:10.1029/93JA02582, 1993.

Harris, E. G.: On a plasma sheath separating regions of oppositely directed magnetic field, Il Nuovo Cimento (1955-1965), 23, 115-121, 1962.

Hayakawa, H., Nishida, A., Hones Jr., E. W., and Bame, S. J.: Statistical characteristics of plasma flow in the magnetotail, J. Geophys. Res., 87, 277-283, 1982.

Hsu, T.-S. and McPherron, R. L.: A statistical study of the relation of Pi2 and plasma flows in the tail, J. Geophys. Res., 112, A05209, doi:10.1029/2006JA011782, 2007.

Huang, C. Y. and Frank, L. A.: A statistical study of the central plasma sheet - Implications for substorm models, Geophys. Res. Lett., 13, 652-655, 1986.

Keiling, A., Angelopoulos, V., Runov, A., Weygand, J., Apatenkov, S. V., Mende, S., McFadden, J., Larson, D., Amm, O., Glassmeier, K.-H., and Auster, H. U.: Substorm current wedge driven by plasma flow vortices: THEMIS observations, J. Geophys. Res.-Space, 114, A00C22, doi:10.1029/2009JA014114, 2009.

Kepko, L., Kivelson, M. G., and Yumoto, K.: Flow bursts, braking, and Pi2 pulsations, J. Geophys. Res., 106, 1903-1916, 2001.

Kepko, L., McPherron, R. L., Amm, O., Apatenkov, S., Baumjohann, W.,Birn, J., Lester, M., Nakamura, R., Pulkkinen, T. I., and Sergeev, V.: Substorm Current Wedge Revisited, Space Sci. Rev., 190, 1-46, 2015.

Kim, H.-S., Lee, D.-Y., Ohtani, S.-I., Lee, E.-S., and Ahn, B.-H.: Some statistical properties of flow bursts in the magnetotail, J. Geophys. Res., 115, A12229, doi:10.1029/2009JA015173, 2010.

Liu, J., Angelopoulos, V., Runov, A., and Zhou, X.-Z.: On the current sheets surrounding dipolarizing flux bundles in the magnetotail: The case for wedgelets, J. Geophys. Res.-Space, 118, 20002020, 2013.

Lucek, E. A., Dunlop, M. W., Balogh, A., Cargill, P., Baumjohann, W., Georgescu, E., Haerendel, G., and Fornacon, K.-H.: Mirror mode structures observed in the dawn-side magnetosheath by Equator-S, Geophys. Res. Lett., 26, 2159-2162, 1999a.
Lucek, E. A., Dunlop, M. W., Balogh, A., Cargill, P., Baumjohann, W., Georgescu, E., Haerendel, G., and Fornacon, K.-H.: Identification of magnetosheath mirror modes in Equator-S magnetic field data, Ann. Geophys., 17, 1560-1573, 1999 b.

Marquardt, D. W.: An Algorithm for Least-Squares Estimation of Nonlinear Parameters, J. Soc. Ind. Appl. Math., 11, 431-441, 1963.

McFadden, J., Carlson, C., Larson, D., Bonnell, J., Mozer, F., Angelopoulos, V., Glassmeier, K.-H., and Auster, U.: THEMIS ESA First Science Results and Performance Issues, Space Sci. Rev., 141, 477-508, 2008.

Nakamura, R., Baumjohann, W., Schödel, R., Brittnacher, M., Sergeev, V. A., Kubyshkina, M., Mukai, T., and Liou, K.: Earthward flow bursts, auroral streamers, and small expansions, J. Geophys. Res., 106, 10791-10802, 2001.

Ohtani, S., Creutzberg, F., Mukai, T., Singer, H., Lui, A. T. Y., Nakamura, M., Prikryl, P., Yumoto, K., and Rostoker, G.: Substorm onset timing: The December 31, 1995, event, J. Geophys. Res., 104, 22713-22728, 1999.

Ohtani, S.-I., Shay, M. A., and Mukai, T.: Temporal structure of the fast convective flow in the plasma sheet: Comparison between observations and two-fluid simulations, J. Geophys. Res.-Space, 109, A03210, doi:10.1029/2003JA010002, 2004.

Pontius Jr., D. H. and Wolf, R. A.: Transient flux tubes in the terrestrial magnetosphere, Geophys. Res. Lett., 17, 49-52, 1990.

Runov, A., Angelopoulos, V., Zhou, X.-Z., Zhang, X.-J., Li, S., Plaschke, F., and Bonnell, J.: A THEMIS multicase study of dipolarization fronts in the magnetotail plasma sheet, J. Geophys. Res.-Space, 116, 517-525, doi:10.1016/j.pss.2010.06.006, 2011.

Runov, A., Angelopoulos, V., Gabrielse, C., Liu, J., Turner, D. L., and Zhou, X.-Z.: Average thermodynamic and spectral properties of plasma in and around dipolarizing flux bundles, J. Geophys. Res.-Space, 120, 4369-4383, 2015.

Schmid, D., Volwerk, M., Plaschke, F., Vörös, Z., Zhang, T. L., Baumjohann, W., and Narita, Y.: Mirror mode structures near Venus and Comet P/Halley, Ann. Geophys., 32, 651-657, doi:10.5194/angeo-32-651-2014, 2014.

Schmid, D., Nakamura, R., Plaschke, F., Volwerk, M., and Baumjohann, W.: Two states of magnetotail dipolarization fronts: A statistical study, J. Geophys. Res., 120, 1096-1108, 2015.

Schödel, R., Baumjohann, W., Nakamura, R., Sergeev, V. A., and Mukai, T.: Rapid flux transport in the central plasma sheet, J. Geophys. Res.-Space, 106, 301-314, 2001.

Sergeev, V. A., Angelopoulos, V., Gosling, J. T., Cattell, C. A., and Russell, C. T.: Detection of localized, plasma-depleted flux tubes or bubbles in the midtail plasma sheet, J. Geophys. Res., 101, 10817-10826, 1996.

Sergeev, V. A., Angelopoulos, V., and Nakamura, R.: Recent advances in understanding substorm dynamics, Geophys. Res. Lett., 39, L05101, doi:10.1029/2012GL050859, 2012.

Sharma, A. S., Nakamura, R., Runov, A., Grigorenko, E. E., Hasegawa, H., Hoshino, M., Louarn, P., Owen, C. J., Petrukovich, A., Sauvaud, J.-A., Semenov, V. S., Sergeev, V. A., Slavin, J. A., Sonnerup, B. U. Ö., Zelenyi, L. M., Fruit, G., Haaland, S., Malova, H., and Snekvik, K.: Transient and localized processes in the magnetotail: a review, Ann. Geophys., 26, 955 1006, doi:10.5194/angeo-26-955-2008, 2008. 
Shiokawa, K., Baumjohann, W., and Haerendel, G.: Braking of high-speed flows in the near-Earth tail, Geophys. Res. Lett., 24, 1179-1182, 1997.

Sonnerup, B. U. O. and Cahill, Jr., L. J.: Magnetopause Structure and Attitude from Explorer 12 Observations, J. Geophys. Res., 72, 171-183, 1967.

Wang, T., Cao, J., Fu, H., Meng, X., and Dunlop, M.: Compressible turbulence with slow-mode waves observed in the bursty bulk flow of plasma sheet, Geophys. Res. Lett., 43, 1854-1861, doi:10.1002/2016GL068147, 2016.

Yao, Z. H., Pu, Z. Y., Fu, S. Y., Angelopoulos, V., Kubyshkina, M., Xing, X., Lyons, L., Nishimura, Y., Xie, L., Wang, X. G., Xiao, C. J., Cao, X., Liu, J., Zhang, H., Nowada, M., Zong, Q. G., Guo, R. L., Zhong, J., and Li, J. X.: Mechanism of substorm current wedge formation: THEMIS observations, Geophys. Res. Lett., 39, L13102, doi:10.1029/2012GL052055, 2012.
Yao, Z. H., Liu, J., Owen, C. J., Forsyth, C., Rae, I. J., Pu, Z. Y., Fu, H. S., Zhou, X.-Z., Shi, Q. Q., Du, A. M., Guo, R. L., and Chu, X. N.: A physical explanation for the magnetic decrease ahead of dipolarization fronts, Ann. Geophys., 33, 1301-1309, doi:10.5194/angeo-33-1301-2015, 2015.

Zhang, L. Q., Liu, Z. X., Baumjohann, W., Ma, Z. W., Pu, Z. Y., Dunlop, M. W., Lu, L., Shi, J. K., Carr, C., Remè, H., and Wang, J. Y.: Convective bursty flows in the nearEarth magnetotail inside $13 R_{\mathrm{E}}$, J. Geophys. Res., 114, A02202, doi:10.1029/2008JA013125, 2009. 